Am J Orthod Dentofacial Orthop. 2013 June ; 143(6): 793-798. doi:10.1016/j.ajodo.2013.01.014.

\title{
Changes in the pattern of patients receiving surgical-orthodontic treatment
}

\author{
William R. Proffit ${ }^{\mathrm{a}}$ [Kenan Distinguished Professor], Tate H. Jackson ${ }^{\mathrm{b}}$ [Postdoctoral \\ fellow], and Timothy A. Turvey ${ }^{\mathrm{C}}$ [Professor and chairman] \\ aDepartment of Orthodontics, School of Dentistry, University of North Carolina, Chapel Hill, NC. \\ bDepartment of Orthodontics, School of Dentistry, University of North Carolina, Chapel Hill, NC. \\ 'Department of Oral and Maxillofacial Surgery, School of Dentistry, University of North Carolina, \\ Chapel Hill, NC.
}

\section{Abstract}

Introduction-The characteristics of patients who seek and accept orthognathic surgery appear to be changing over time but have not been well documented in the 21 st century.

\begin{abstract}
Methods-Records for patients who had orthognathic surgery at the University of North Carolina from 1996 to 2000 and from 2006 to 2010 were reviewed to collect data for changes in the prevalence of patients with mandibular deficiency (Class II), maxillary deficiency or mandibular prognathism (Class III), long face, and asymmetry problems. The changes were compared with those in previous time periods and at other locations.
\end{abstract}

\begin{abstract}
Results-Between 1996 and 2000 and between 2006 and 2010, the percentage of Class III patients increased from 35\% to 54\%, and the percentage of Class II patients decreased from 59\% to $41 \%$, while the percentages for long face and asymmetry showed little change. The decrease in Class II patients was accentuation of a long-term trend; the increase in Class III patients occurred only after the turn of the century.
\end{abstract}

Conclusions-A similar but less-marked change has been noted at some but not all other locations in the United States. It appears to be related primarily to an increase in the numbers of African Americans, Native Americans, Hispanics, and Asians who now are seeking surgical treatment, but it also has been affected by changes in where orthognathic surgery is performed, decisions by third-party payers (insurance and Medicaid) about coverage for treatment, and the availability of nonsurgical orthodontic treatment options for Class II patients.

The characteristics of patients who seek evaluation for dentofacial problems that might require orthognathic surgery for effective treatment, and the characteristics of those who accept surgery, are needed in planning the management of this patient group. Using data from the National Center for Health Statistics that were published in the $1970 \mathrm{~s},{ }^{1}$ we

Copyright $(9) 2013$ by the American Association of Orthodontists.

Reprint requests to: William R. Proffit, Department of Orthodontics, UNC School of Dentistry, Chapel Hill, NC 27599-7450; William_Proffit@dentistry.unc.edu.

The authors report no commercial, proprietary, or financial interest in the products or companies described in this article. 
evaluated the number of patients who would be expected to need surgical-orthodontic treatment based on the severity of their malocclusion ${ }^{2}$ and compared this with the percentage who sought treatment by making appointments for evaluation in the Dentofacial Clinic at the University of North Carolina (Fig 1). ${ }^{3}$ We estimated that the most severe $5 \%$ of all Class II patients and the most severe $33 \%$ of Class III patients were potential candidates for orthognathic surgery. It was particularly difficult to determine treatment needs for longface patients from the data for open-bite prevalence, because although two thirds of this group had an anterior open bite, a third did not. The prevalence of long-face problems was calculated by assuming that an open bite greater than $3 \mathrm{~mm}$ indicates a skeletal long-face problem; we hoped that false negatives (long face but no open bite) would be balanced by false positives (open bite but normal face height) and estimated that $25 \%$ of this group would need surgery. By using these estimates, the data showed that patients with Class II malocclusion were underrepresented in the group seeking treatment, whereas Class III patients were modestly overrepresented, and open-bite patients were greatly overrepresented (Fig 1).

The most recent study of the prevalence of various types of malocclusion in the United States (National Health and Nutrition Examination Survey [NHANES] III, published in the 1990s) provides data for types of malocclusion by severity of the deviations from normal. ${ }^{4}$ Figures 2 and 3, calculated from the NHANES III data, show the prevalence for overjet and incisor overlap, respectively. For both, it is reasonable to expect that essentially all extreme deviations and some severe deviations from ideal relationships would lead to a need for orthognathic surgery. The NHANES data also show that the prevalence of extreme reverse overjet (Class III) is 3 times higher in Asians than in blacks or whites, and twice as great in Hispanics (Mexican Americans). Extreme overjet (Class II) is twice as prevalent in black and Hispanic people as in white people. Extreme open bite (long face) is 7 times more prevalent in African Americans than in whites and is almost never observed in Hispanics. Dental and facial asymmetry were not evaluated in the NHANES III.

The NHANES III data made it possible to calculate what percentages of the US population would fall into the grades in the index of treatment need (IOTN) that was developed in the United Kingdom in the 1980s to help in the selection of patients for orthodontic treatment in the National Health Service. We did this for the 3 major racial-ethnic groups (white, black, Hispanic [Mexican-American]) that were included in the NHANES III. ${ }^{5}$ The prevalence of IOTN grades 4 and 5 (severe) is much greater in blacks than in whites or Hispanics, whereas mild problems (IOTN grade 2) are more frequent in whites than in blacks and much more frequent than in Hispanics (Fig 4). As Figure 4 also shows, from 1989 to 1991, orthodontic treatment was much more likely to be received by white people than either of the other groups. The extent to which that has changed since then has not been documented.

The change in the prevalence of skeletal Class I, Class II, and Class III in the population who sought evaluation in the dentofacial program at the University of North Carolina over a 20-year span up to the mid-1990s is shown in Figure 5. ${ }^{6}$ In this data set, most of the Class I patients were seeking surgical correction of facial asymmetry, but a few long-face patients who had no anteroposterior problem were included. There was a modest but steady decline 
in Class II patients, with fluctuations but no long-term trend toward change in the prevalence of Class III.

Has the distribution of patients seeking and receiving surgical treatment changed since the turn of the 21st century? Our clinical impression was that both the number and proportion of Class III patients have greatly increased; to evaluate this, we compared the characteristics of surgery patients at the University of North Carolina in 1996 through 2000 with those in 2006 through 2010.

\section{MATERIAL AND METHODS}

The database for our dentofacial program includes all patients who were referred for evaluation through the Dentofacial Clinic, and all patients who had orthognathic surgery here, whether or not their initial evaluation was here. For this study, the primary source was data for the patients who actually had surgery, not those who were evaluated as potential surgery patients. Records for all patients who had surgery between 1996 and 2000 and between 2006 and 2010 were reviewed to confirm the jaw movements at surgery, because this sometimes changes from the pretreatment plan. The 2 periods 10 years apart were selected to sharpen the contrast between them. About half of the patients for whom a surgical plan was recommended did not have the surgery. A major reason for not accepting treatment was difficulty in obtaining approval for health insurance coverage for surgery and hospitalization.

Patients who had mandibular advancement with or without other surgical procedures were the Class II group, and those who had maxillary advancement, mandibular setback, or a combination of these procedures were the Class III group. The Class II and Class III groups were mutually exclusive. Patients who had the maxilla moved up more than $2 \mathrm{~mm}$, alone or combined with other directions of maxillary repositioning or with mandibular surgery, were the long-face group, and those who had maxillary or mandibular surgery to correct asymmetry were the asymmetry group. These groups did overlap with the Class II and Class III groups and with each other.

\section{RESULTS}

Sex, racial or ethnic, and age characteristics of the 2 sample groups are shown in the Table. In both periods, most of the patients were white, but this decreased from 88\% in 1996 to 2000 to $76 \%$ in 2006 to 2010. The percentage of African Americans nearly doubled, from $6 \%$ to $11 \%$, but because the 2010 US census showed that they comprise $22 \%$ of the population of North Carolina, they still are underrepresented. The number of Hispanic patients increased from $1 \%$ to $3 \%$, coming closer to their percentage of the state population $(8.4 \%)$, but they also remain underrepresented. Interestingly, the percentage of Asian patients grew to parity with their prevalence in the state population $(2.2 \%)$, whereas the increase in the number of Native Americans who had surgery made them slightly overrepresented relative to their prevalence in the population of North Carolina at large (1.3\% in 2010). ${ }^{7}$ 
The percentages of patients having surgery for Class II correction, Class III, long-face problems, and asymmetry correction are presented graphically in Figure 6. The expected percentages, except for asymmetry, are the same as those calculated previously and shown in Figure 1. For the US population as a whole, over $80 \%$ have some degree of facial asymmetry, but this is perceived as a problem only when it is great enough to attract attention, and there are no good data for degrees of asymmetry that do create a problem, so the 20\% expected number used in Figure 6 is only a rough estimate.

Note that the percentages for Class II and Class III almost reversed in 2006 to 2010 from what they were in 1996 to 2000, with only $41 \%$ Class II and 54\% Class III patients in the more recent group $(P<0.0001$; Fisher exact test). The number of long-face patients and those requiring asymmetry correction remained relatively steady over the total duration of the survey.

\section{DISCUSSION}

Two questions immediately come to mind as the changes at the University of North Carolina are considered: (1) is this just a phenomenon here and (2) what are the possible explanations for the change?

It is difficult to be sure whether this change has occurred only in North Carolina, because there are no published longitudinal data for other locations, and release of data from other American centers now is inhibited by concern about privacy regulations and problems in institutional review board approval for using patient data for studies outside that center. A national prevalence estimate comes from the 2008 nationwide inpatient sample. ${ }^{8}$ This report was based on queries to representative hospitals throughout the United States regarding patient admissions and treatments rendered. Analysis of diagnostic codes for maxillofacial procedures showed that in $2008,2.2 \%$ of all hospitalized patients were operated on with a diagnosis consistent with Class III malocclusion, whereas only $1.4 \%$ were Class II. ${ }^{8}$ Of the patients who had orthognathic surgery, $30 \%$ had either maxillary hyperplasia or mandibular hypoplasia corrected (Class II indicators), and $47 \%$ had mandibular hyperplasia or maxillary hypoplasia corrected (Class III indicators). Diagnosis of jaw hyperplasia or hypoplasia to infer skeletal malocclusion must be used with caution, however, since deviations in jaw size can occur in all 3 planes of space. Nevertheless, this national prevalence estimate aligns well with our recent data at the University of North Carolina. To determine whether orthognathic surgery in North Carolina is simply moving toward an already established norm or mirrors a national shift in care would require longitudinal national data.

We requested data for Class III vs Class II surgery in recent years from many other surgical centers; it was difficult to obtain because of concerns about Health Insurance Portability and Privacy Act (HIPPA) restrictions and informed consent. We obtained information only from the San Antonio Military Medical Center, the surgical practices in Alabama that handled surgeries from 1 orthodontic office, and the Department of Oral and Maxillofacial Surgery at the University of Washington in Seattle. 
In a sense, the military population represents a national sample because military personnel come from all parts of the country, although the percentage of young African American adults in the military is somewhat larger than in the general population. From July 2010 through June 2012, the military's San Antonio center had 72 Class II patients and 78 Class III patients, a higher percentage of Class III relative to Class II than the University of North Carolina had in the late 1990s, but fewer than here more recently.

Data collected from Birmingham, Alabama, from 2000 to $2002(\mathrm{n}=109)$ and from 2010 to $2012(\mathrm{n}=100)$ show a significant decrease in the number of treated Class II patients from $64 \%$ to $40 \%$, and a minimal increase in the proportion of treated Class III patients from $31 \%$ to $34 \%$. Interestingly, the proportion of Class I patients increased significantly from 5\% to $26 \%$ over this same 10-year time span, with most of this group treated with 2-jaw surgery to rotate the occlusal plane or with bimaxillary advancement to control sleep apnea. Although these results do not mirror the increase in Class III patients seen at the University of North Carolina, they do confirm the relative decrease in Class II patients undergoing treatment over the past decade in this region of the country.

Data from the Department of Oral and Maxillofacial Surgery at the University of Washington collected in $2009(\mathrm{n}=160)$ and during the first 6 months of $2012(\mathrm{n}=102)$ show that about a third of patients operated on during that time had Class III problems; this proportion is similar to the percentage at the University of North Carolina a decade previously.

Using data from a 3-year recall of 516 patients who had orthognathic surgery in Oslo, Norway, between 1994 and 2002, Espeland et $\mathrm{al}^{9}$ noted that $48 \%$ of the female patients and $64 \%$ of the male patients had treatment for a Class III problem, whereas $36 \%$ of the females and $23 \%$ of the males had Class II treatment. Since skeletal Class III is much less frequent in Scandinavia than skeletal Class II, it is apparent that the Class III patients were relatively overrepresented. The extent to which that has changed in Norway in recent years has not been reported. In a Brazilian sample of orthognathic surgery patients in the Araraquara, S ao Paulo, area treated between 2000 and 2006, 47\% had Class III and 46\% had Class II surgery. ${ }^{10}$ The prevalence of Class II vs Class III problems in that population and any changes over time were not reported.

We asked why these changes have occurred at the University of North Carolina. The increased numbers of African American, Hispanic, Native American, and Asian patients having orthognathic surgery here certainly could have contributed to the increase in Class III surgeries in recent years, and appears to have done so. As we have noted, all of these groups have higher percentages of Class III malocclusion than do white people, particularly those of northern European origin. But these groups were 19\% of the total, and the Class III percentage jumped from $34 \%$ to $54 \%$, so even if every one of them had been Class III (not the case), this would not totally account for the change. In fact, $69 \%$ of the 135 minority patients had Class III surgery, 28\% had Class II surgery, and 3\% had Class I (asymmetry) surgery. Similarly, the decrease in Class II patients who had surgery at the University of North Carolina cannot be explained entirely by the smaller percentage of white patients. 
Several other factors could have contributed to this change, unfortunately with no good quantitative data to help evaluate their importance.

There could be psychosocial effects. In a general population largely of European origin, some degree of mandibular deficiency and a convex profile outline often is observed. It seems reasonable that this is more socially acceptable than mandibular excess or maxillary deficiency that produces a concave profile. A study published in 2002 with a European sample concluded that patients with a Class III malocclusion were significantly more likely to report feeling unattractive preoperatively than were Class II patients. ${ }^{11}$ The long-face pattern of deformity that usually accompanies severe open bite also is less socially acceptable. The numbers of long-face and asymmetry patients have changed only slightly in our samples, if at all. Is it possible that a Class III skeletal pattern now is less acceptable, and a Class II skeletal pattern more acceptable, than only a few years ago?

It is widely perceived that a prominent (strong) chin is more masculine and indicates more aggressive behavior than does a retrusive (weak) chin, and softening the facial appearance is a major reason for surgery in many Class III patients, especially women. A decrease in the number of Class II patients also would be compatible with the idea that a less aggressive facial appearance is more desirable now. Our data, however, show that patients are more likely to accept Class III surgery when their primary problem is maxillary deficiency, so facial concavity also plays a role in the decision.

Asymmetry frequently accompanies disturbances in mandibular growth and is perceived as a problem if it is severe. Of the patients evaluated in our Dentofacial Clinic in the 1990s, $42 \%$ of the Class III and long-face patients had noticeable facial asymmetry, predominantly in chin position. The Class II patients were less asymmetric, with a $28 \%$ prevalence. ${ }^{12}$ Even if one presumes that asymmetry now also is less socially acceptable, and that the higher prevalence of asymmetry in the Class III group is a factor in more patients receiving surgery now, the difference probably explains only a small part of the recent change.

There have also been changes in nonsurgical treatment options. Modern orthodontic techniques in the United States include many options for correcting Class II malocclusions without relying on patient compliance. The increased use of both skeletal anchorage and tooth-borne appliances (eg, the Herbst appliance in adults) to camouflage large Class II skeletal relationships might help explain the proportional decrease in Class II patients seeking surgery. ${ }^{13}$ Fewer noncompliant treatment modalities to compensate for Class III skeletal relationships are available, and the unpredictable nature of late mandibular growth in this group also affects the probable success of orthodontic camouflage. Perhaps the changes we have seen at the University of North Carolina are, in part, a reflection of changes in the alternatives to surgical treatment.

Referral patterns and insurance coverage are also different now. In North Carolina, as in many states, fewer patients now have orthognathic surgery in private practices, and more patients (especially those with minimal or no insurance) are referred to an academic medical center. This has led to a considerable increase in the number of surgery patients added to our database, from 287 in 1996 through 2000 to 560 in 2006 through 2010. Medicaid patients in 
North Carolina largely can have surgical treatment only at the University of North Carolina, and this also probably affected the racial and ethnic aspect of the patient pool. African Americans and Hispanics are more likely to be covered by Medicaid than are white people or the other minority groups.

With a larger proportion of patients treated at the University of North Carolina now having Medicaid coverage, public policy might have influenced the concurrent changes in treatment patterns. The criteria in North Carolina that dictate which patients qualify for Medicaid coverage for orthognathic surgery emphasize functional deficits and medical necessity, often due to tissue damage ${ }^{14}$ Patients with Class III jaw relationships could be more likely to be viewed as having functional issues, since they often cannot posture their jaws to create anterior occlusion and have functional shifts caused by either anterior or posterior (or both) crossbites.

The spectrum of treatment available might affect the likelihood of insurance coverage for surgery as well. Surgery might be viewed as medically necessary when it is the only way to correct a severe malocclusion. For example, insurance companies might not approve a mandibular osteotomy to correct extreme overjet in a Class II patient under the assumption that no matter how severe, it can be corrected with skeletal anchorage and orthodontic treatment. Accordingly, insurance policies, whether public or private, might have had a significant effect on the changes we have seen.

\section{CONCLUSIONS}

Compared with a decade ago, a greater proportion of patients undergoing orthognathic surgery at the University of North Carolina have Class III rather than Class II problems. Although the changing demographics of those who seek surgical correction of severe malocclusion has most likely contributed most to this development, changes in nonsurgical orthodontic treatment options, shifting patterns in where surgery is performed, and changes in who pays for treatment have probably all played roles in this trend.

\section{Acknowledgments}

We thank Curtis Marsh, David Sarver, and Dale Bloomquist for data for the procedures in their locations; Debora Price for extracting and organizing the data from the dentofacial database at the University of North Carolina; and Ceib Phillips for the statistical analysis.

Supported in part by NIH grants DE-05215 and T90DE-021986-01 from the National Institute of Dental and Craniofacial Research.

\section{REFERENCES}

1. Kelly, J.; Harvey, C. An assessment of the teeth of youths 12-17 years. Washington, DC: National Center for Health Statistics; 1977. (DHEW publication no. [HRA] 77-1644).

2. Proffit WR, White RP Jr. Who needs surgical-orthodontic treatment? Int J Adult Orthod Orthognath Surg. 1990; 5:81-90.

3. Proffit WR, Phillips C, Dann CIV. Who seeks surgical-orthodontic treatment? The characteristics of patients evaluated in the UNC dentofacial clinic. Int J Adult Orthod Orthognath Surg. 1990; 5:153160 . 
4. Brunelle JA, Bhat M, Lipton JA. Prevalence and distribution of selected occlusal characteristics in the US population, 1988-91. J Dent Res. 1996; 75:706-713. [PubMed: 8594094]

5. Proffit WR, Fields HW, Moray LJ. Prevalence of malocclusion and orthodontic treatment need in the United States: estimates from the NHANES III survey. Int J Adult Orthod Orthognath Surg. 1998; 13:97-106.

6. Bailey LJ, Haltiwanger LH, Blakey GH, Proffit WR. Who seeks surgical-orthodontic treatment: a current review. Int J Adult Orthod Orthognath Surg. 2001; 16:280-292.

7. [Accessed on September 15] 2010 census demographic profile summary file prepared by the U.S. Census Bureau, 2011. 2012. Available at: http://quickfacts.census.gov/qfd/states/37000.html.

8. Venugoplan SR, Nanda V, Turkistani K, Desai S, Allareddy V. Discharge patterns of orthognathic surgeries in the United States. J Oral Maxcillofac Surg. 2012; 70:e77-e86.

9. Espeland L, Høgevold HE, Stenvik A. A 3-year patient-centred follow-up of 516 consecutively treated orthognathic surgery patients. Eur J Orthod. 2008; 30:24-30. [PubMed: 18033770]

10. Boeck EM, Lunardi N, Pinto Ados DS, Pizzol KE, Boeck Neto RJ. Occurrence of skeletal malocclusions in Brazilian patients with dentofacial deformities. Braz Dent J. 2011; 22:340-345. [PubMed: 21861036]

11. Gerzanic L, Jagsch R, Watzke IM. Psychologic implications of orthognathic surgery in patients with skeletal Class II or Class Ill malocclusion. Int J Adult Orthod Orthognath Surg. 2002; 17:7581.

12. Severt TR, Proffit WR. The prevalence of facial asymmetry in the dentofacial deformities population at the University of North Carolina. Int J Adult Orthod Orthognath Surg. 1997; 12:171176.

13. Ruf S, Pancherz H. Orthognathic surgery and dentofacial orthopedics in adult Class II Division 1 treatment: mandibular sagittal split osteotomy versus Herbst appliance. Am J Orthod Dentofacial Orthop. 2004; 126:140-152. [PubMed: 15316468]

14. North Carolina Division of Medical Assistance: orthodontic services. Medicaid and health choice clincial coverage policy no. 4B; revised. 2012 Mar 12. 


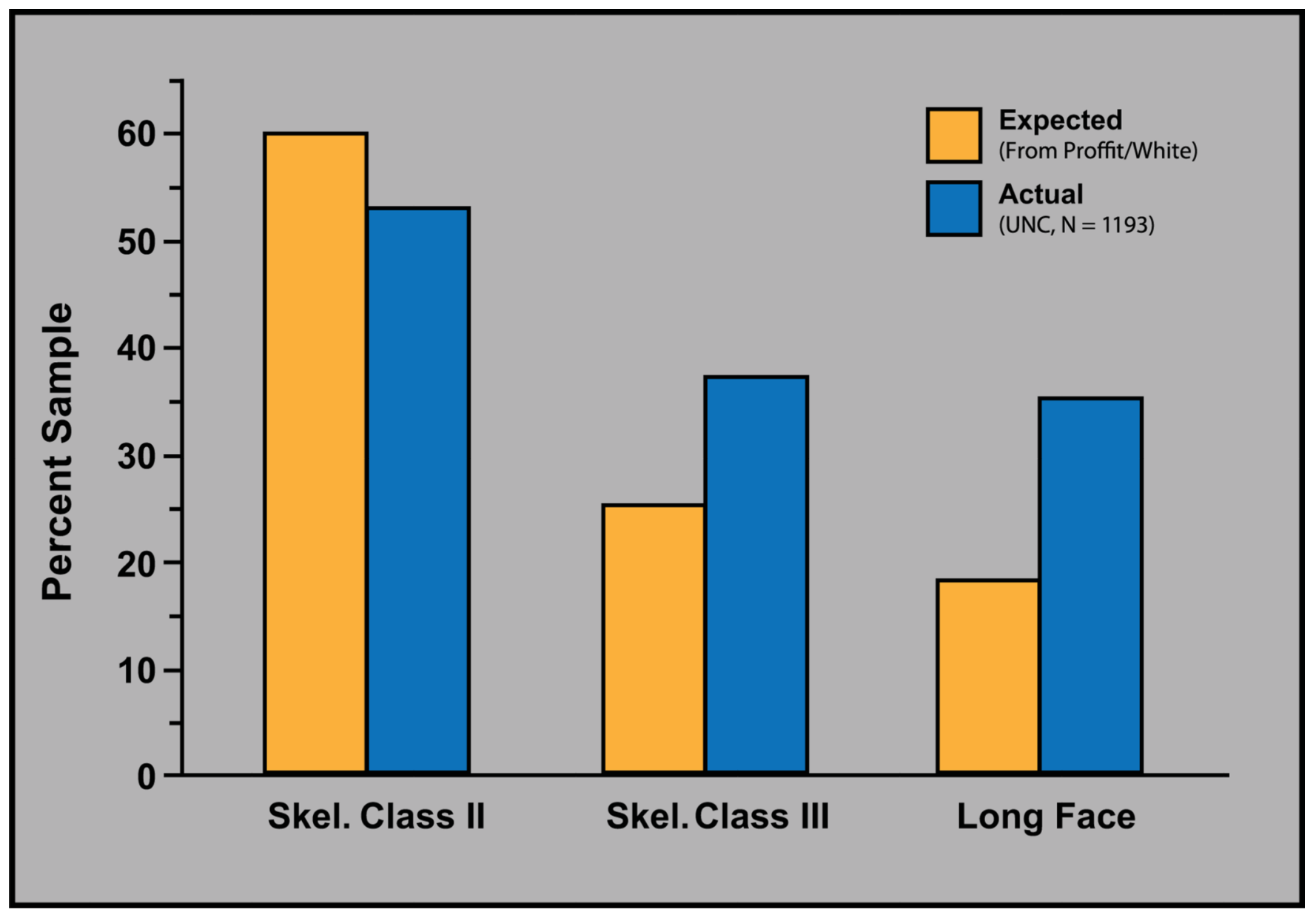

Fig 1.

The expected and actual percentages of patients who sought evaluation in the University of North Carolina Dentofacial Clinic, 1979-1989. Patients with Class II problems (mandibular deficiency) were underrepresented in the clinic population compared with their prevalence in the general population, Class III patients (maxillary deficiency or mandibular prognathism) were overrepresented, and long-face patients were greatly overrepresented. 


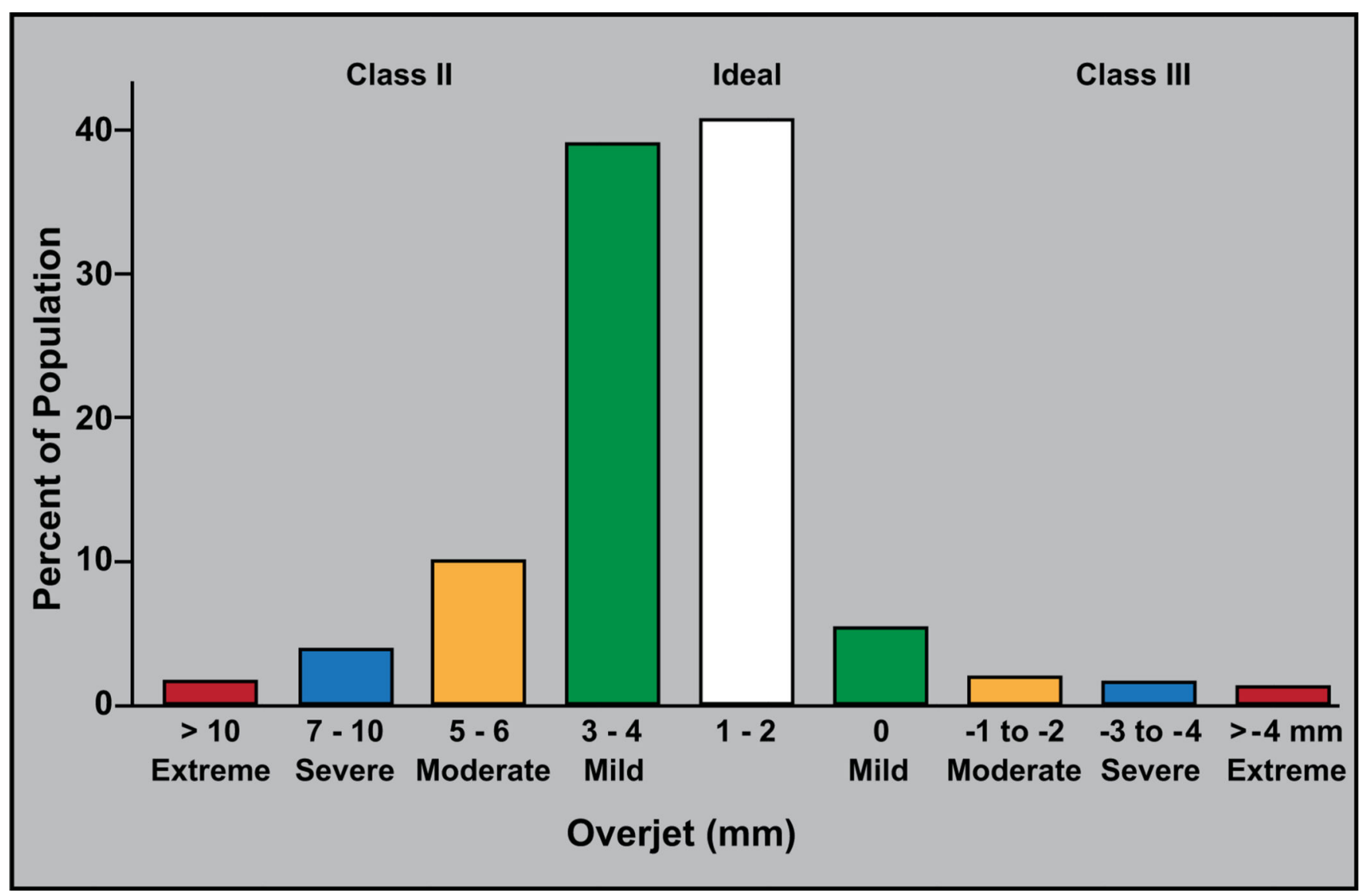

Fig 2.

Overjet (Class II) and reverse overjet (Class III) in the US population, 1989-1991. ${ }^{4}$ Most of those with extreme deviation from the ideal and some but not all with severe deviation would be candidates for orthognathic surgery. 


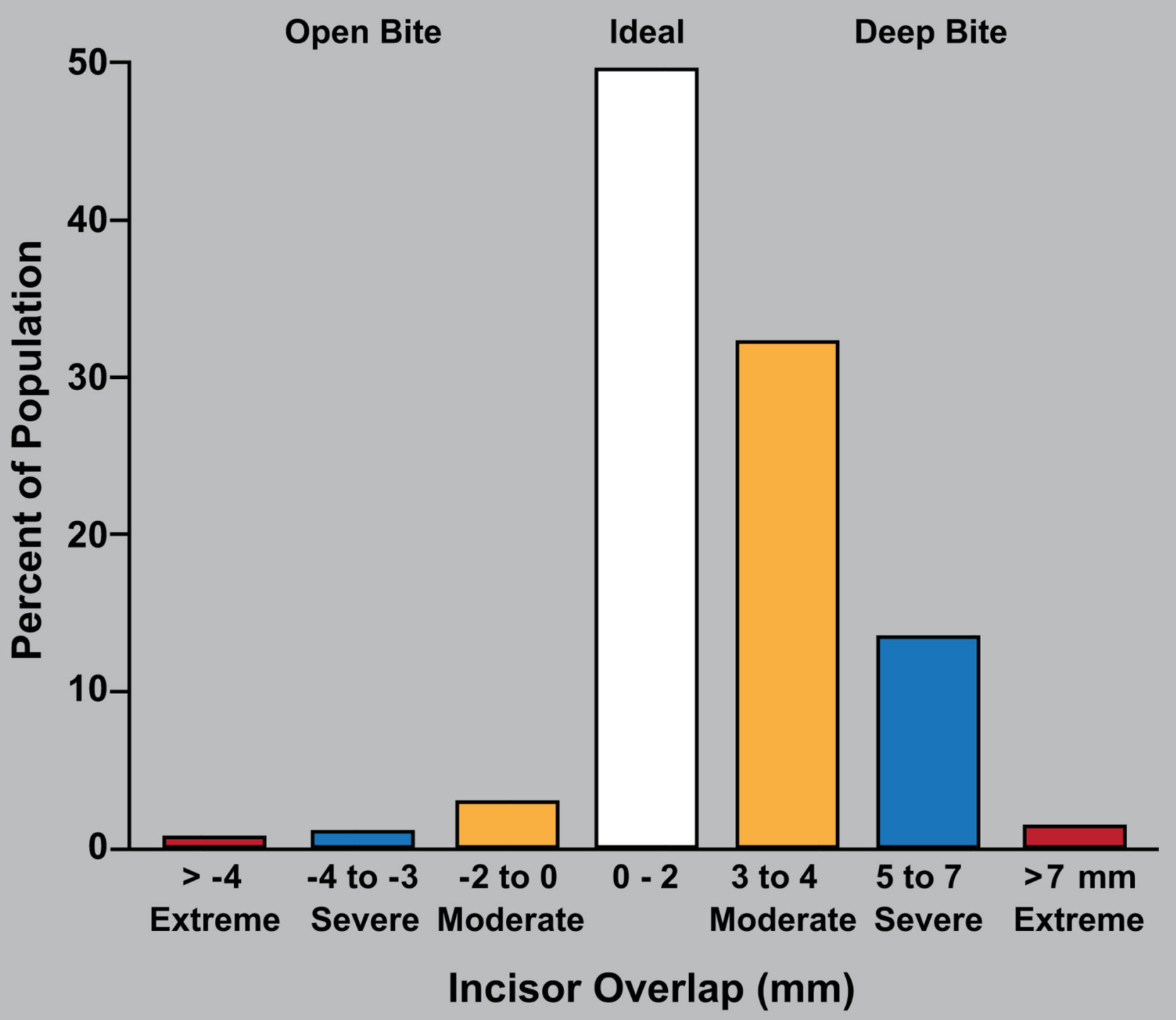

Fig 3.

Open-bite and deepbite relationships in the US population, 1989-1991. ${ }^{4}$ As with overjet, those with the most severe deviation from ideal would be candidates for orthognathic surgery. 


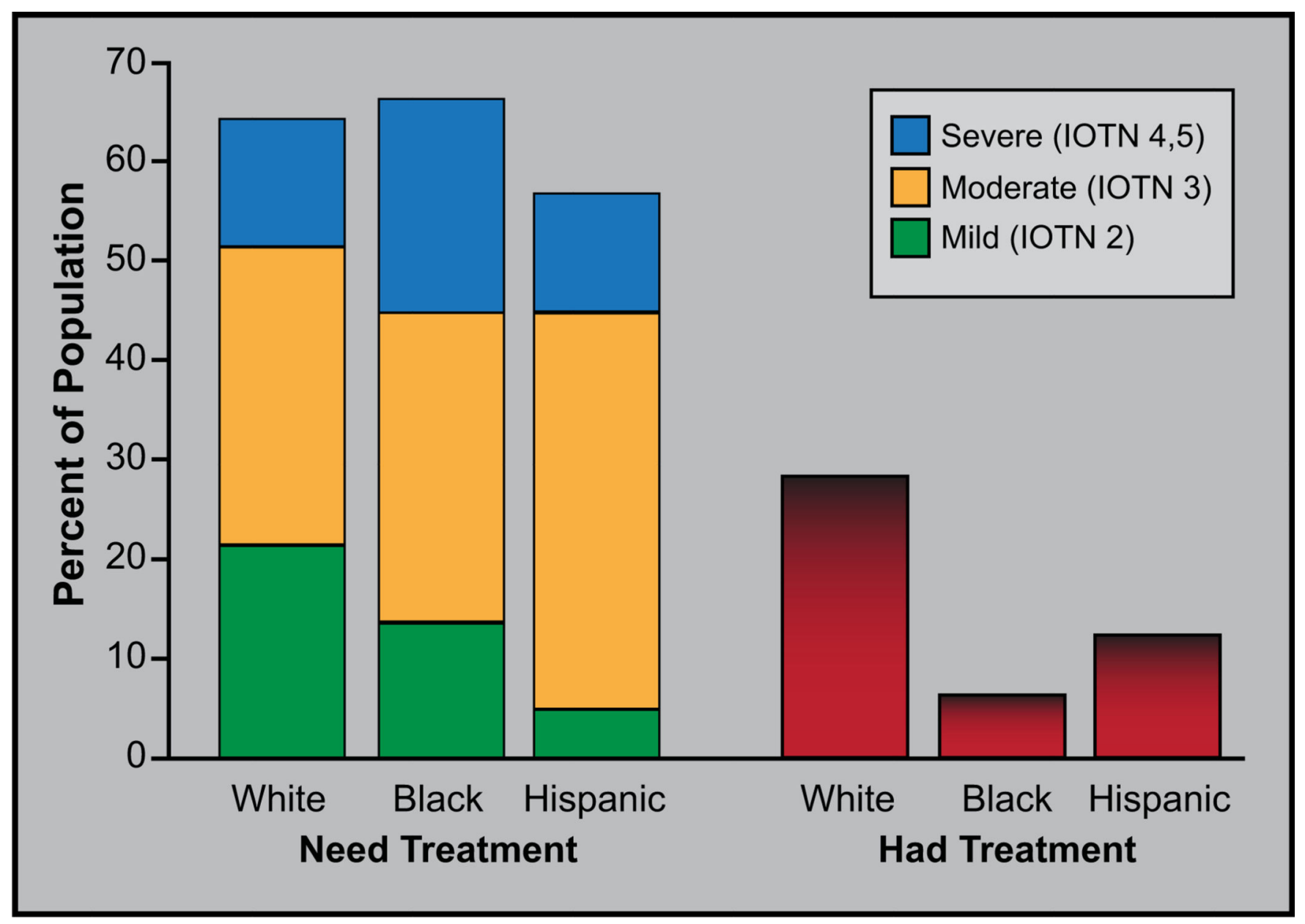

Fig 4.

Orthodontic treatment need by severity of the problem for white, black, and Hispanic (Mexican American) youths aged 12 to 17 years, 1989-1991, and the percentage of each group who reported receiving orthodontic treatment. The prevalence of severe and very severe problems (IOTN grades 4 and 5) was nearly twice as great in blacks than either whites or Hispanics. 


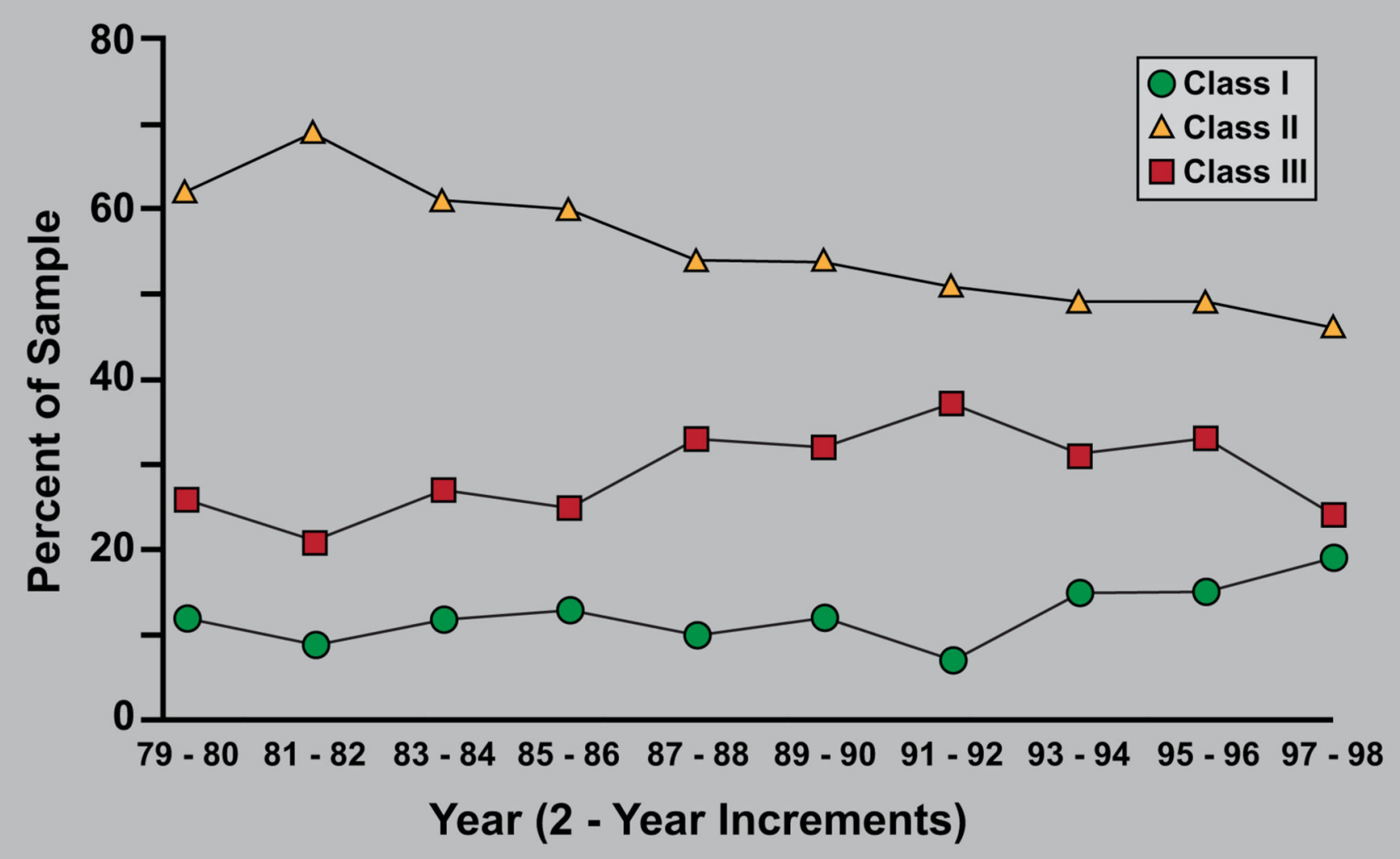

Fig 5.

Data for the prevalence of Class I, Class II, and Class III patients evaluated in the University of North Carolina Dentofacial Clinic in 2-year increments in 1979 and 1980 to 1997 and 1998. Class I patients with no anteroposterior discrepancy were largely seeking correction of asymmetry, but this group also included some long-face patients. The percentage of Class II patients declined slowly but steadily; the percentages of Class III and Class I patients fluctuated but showed no sustained change. 


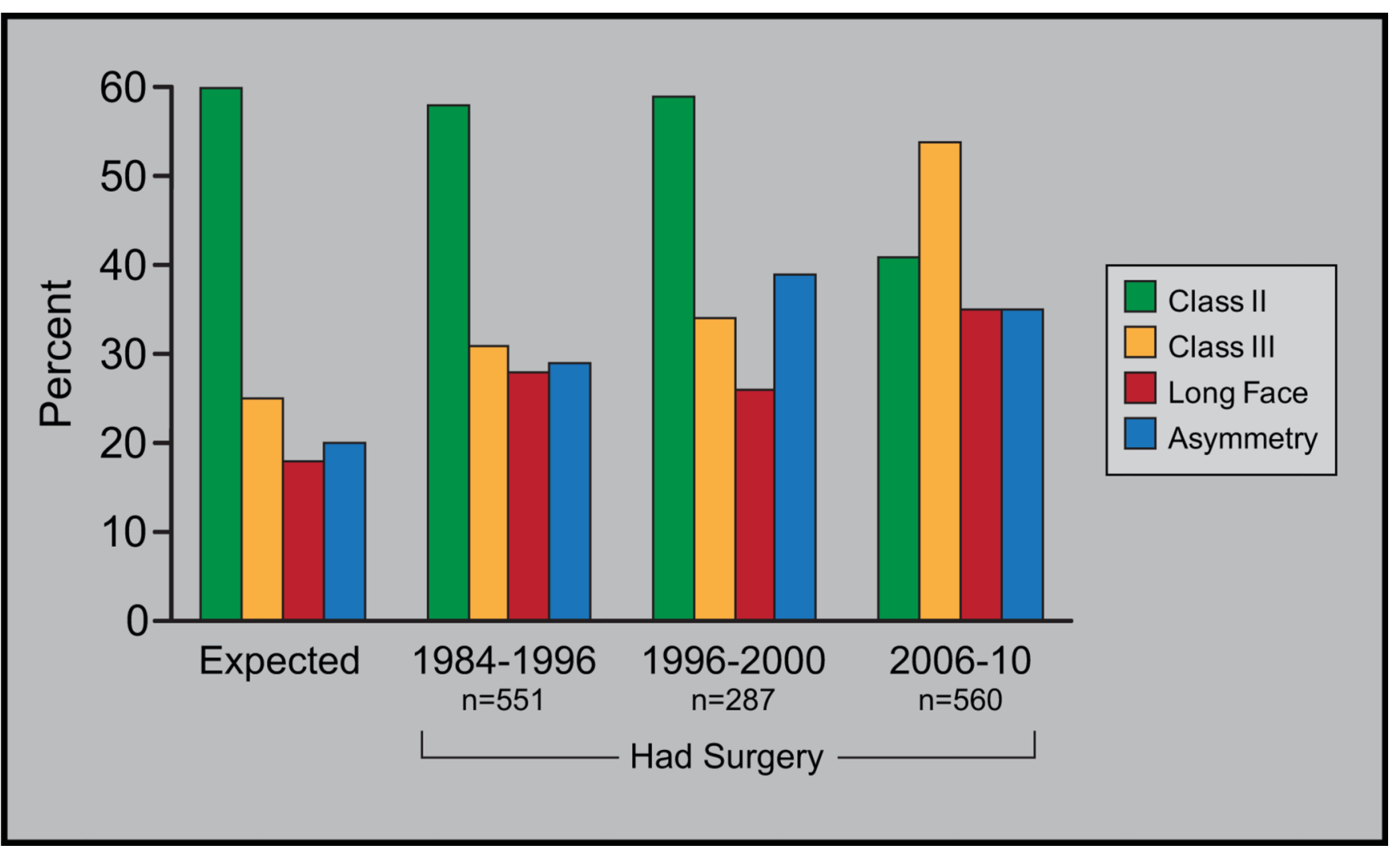

Fig 6.

Expected vs actual percentages of Class II, Class III, long-face, and asymmetry patients having surgery at the University of North Carolina. The data for 1984 through 1996 were taken from the study of Bailey et al. ${ }^{6}$ Note the decline in the percentage of Class II surgeries from 1996 through 2000 to 2006 through 2010, and the increase in Class III. The percentage of long-face patients also increased in the most recent group, and the asymmetry percentage declined slightly. 


\section{Table}

Sex, racial and ethnic, and age characteristics of orthognathic surgery patients

\begin{tabular}{lccccc}
\hline & \multicolumn{2}{c}{$\mathbf{1 9 9 6 - 2 0 0 0}$} & & \multicolumn{2}{c}{$\mathbf{2 0 0 6 - 2 0 1 0}$} \\
\cline { 2 - 3 } \cline { 5 - 6 } & $\mathbf{n}$ & \% & & $\mathbf{n}$ & \% \\
\hline Gender & 304 & & 564 & \\
\hline Female & 196 & 64 & 352 & 62 \\
\hline Race/ethnicity & 283 & & 557 & \\
\hline White & 246 & 87 & 422 & 76 \\
\hline African American & 18 & 6 & 62 & 11 \\
\hline Hispanic & 3 & 1 & 14 & 3 \\
\hline Native American & 1 & 0.4 & 14 & 3 \\
\hline Asian & 1 & 0.4 & 13 & 2 \\
\hline Other & 14 & 5 & 32 & 6 \\
\hline Age & $12-73$ & & $11-58$ & \\
\hline Mean/SD (y) & $23.6 \pm 10.9$ & & $22.2 \pm 9.1$ & \\
\hline Range (y) & & & & & \\
\hline
\end{tabular}

